( ) Д.Н. Лаптев, А.О. Емельянов*, Е.Д. Медведева, С.В. Переверзева, В.А. Петеркова

Национальный медицинский исследовательский центр эндокринологии, Москва

ОБОСНОВАНИЕ. За последние годы помповая инсулинотерапия, также известная как непрерывная подкожная инфузия инсулина (НПИИ), стала распространенным методом лечения детей с сахарным диабетом 1 типа (СД1). Несмотря на то что в целом дети с СД1 на помповой терапии достигают лучших показателей гликемического контроля, при этом отмечается значительная гетерогенность метаболических исходов среди отдельных пациентов, многие дети на НПИИ не достигают целевого уровня $\mathrm{HbA}_{1 c^{\circ}}$

ЦЕЛЬ. Оценить уровень гликемического контроля и факторы, влияющие на эффективность длительного применения НПИИ у детей.

МАТЕРИАЛЫ И МЕТОДЫ. Проанализированы данные специализированного регистра пациентов с СД1, переведенных на помповую инсулинотерапию в возрасте 1-18 лет за более чем 3 года до момента исследования. Оценивались показатели гликированного гемоглобина $\left(\mathrm{HbA}_{1 c}\right)$, частота и факторы, значимо ассоциированные с ответом или прекращением использования НПИИ.

РЕЗУЛЬтАТЫ. Уровень НbА личением числа пациентов, достигнувших целевого уровня $\mathrm{HbA}_{1 c}(<7,5 \%)$, с 17 до 36\%. Лучший ответ отмечался при переводе на помповую инсулинотерапию у пациентов в возрасте до 6 лет, с НbА более 9\%, а также у пациентов, регулярно использующих дополнительные болюсные и базальные функции и непрерывный мониторинг глюкозы. Основная причина прекращения использования инсулиновой помпы - неудобство использования и ношения - 47,7\%. Факторы риска отказа от помпы: более поздний возраст перевода на НПИИ и частые эпизоды тяжелой гипогликемии.

ЗАКЛЮЧЕНИЕ. По результатам проведенного исследования показано, что помповая терапия является эффективным методом инсулинотерапии, который позволяет достигнуть более низкого уровня $\mathrm{HbA}_{1 с}$ по сравнению с исходными значениями.

КЛЮЧЕВЫЕ СЛОВА: сахарный диабет 1 типа; сахарный диабет у детей и подростков; помповая инсулинотерапия; НПИИ; НМГ

\title{
LONG-TERM GLYCEMIC CONTROL AND FACTORS, ASSOCIATED WITH RESPONSE TO PUMP INSULIN THERAPY IN CHILDREN
}

(c) Dmitry N. Laptev, Andrey O. Emelyanov*, Elena D. Medvedeva, Svetlana V. Pereverzeva, Valentina A. Peterkova

Endocrinology Research Centre, Moscow, Russia

BACKGROUND: In recent years, pump-based insulin therapy, also known as continuous subcutaneous insulin infusion (CSII), has become a common treatment for children with type 1 diabetes mellitus (T1DM). Despite the fact that, in general, children with type 1 diabetes achieve the best glycemic control indices during pump therapy, while there is a significant heterogeneity of metabolic outcomes among individual patients, many children with CSII do not reach the target level of $\mathrm{HbA}_{1 c^{*}}$

OBJECTIVE: To assess the level of glycemic control and factors associated by withdrawal of use, the response to treatment with prolonged use of CSII in children with type 1 diabetes.

MATERIALS AND METHODS: The study included 458 children aged 1 to 18 years, treated to pump therapy at least 3 years before the study, the presence of the analyzed data in the register.

RESULTS: The level of $\mathrm{HbA}_{1 c}$ decreased by $-0.7 \%$ compared with the primary endpoint, which was accompanied by an increase in the number of patients who reached the target level of $\mathrm{HbA}_{1 \mathrm{c}}(<7.5 \%)$ from $17 \%$ to $36 \%$. The best response was observed for patients under 6 years of age with $\mathrm{HbA}_{1 c}$ over $9 \%$ for pump insulin therapy, as well as in patients who regularly use additional bolus and basal functions and CGM. The main reason for stopping the use of the insulin pump is the inconvenience of using and wearing - $47.7 \%$. Risk factors for pump abandonment: later age of start treatment on CSII and frequent episodes of severe hypoglycemia.

CONCLUSION: According to the results of the study, it was shown that pump therapy is an effective method of insulin therapy, which allows to achieve a lower level of $\mathrm{HbA}_{1 c}$ compared to the initial values.

KEYWORDS: type 1 diabetes mellitus; diabetes mellitus in children and adolescents; insulin pump therapy; CSII; CGM 
За последние годы помповая инсулинотерапия, также известная как непрерывная подкожная инфузия инсулина (НПИИ), стала распространенным методом лечения детей с сахарным диабетом 1 типа (СД1). Значительному росту использования НПИИ способствовали технологическое совершенствование помп с момента их появления в 1970-х годах и понимание необходимости достижения целевого уровня гликемического контроля для снижения риска развития микро- и макрососудистых осложнений СД1 [1]. Помповая инсулинотерапия имитирует физиологическую секрецию инсулина лучше, чем в случае с множественными инъекциями инсулина (МИИ), и дает пациентам большую гибкость при приеме пищи и физической активности $[2,3]$. В нескольких метаанализах рандомизированных клинических исследований показано, что использование НПИИ у детей с СД1 сопровождается более низкими показателями $\mathrm{HbA}_{1 с}$ по сравнению с МИИ $[4,5]$.

Несмотря на то что в целом дети с СД1 на помповой терапии достигают лучших показателей гликемического контроля, отмечается значительная гетерогенность метаболических исходов среди отдельных пациентов. Вместе с тем результаты проведенных ранее исследований указывают на то, что часть детей на НПИИ не достигают целевого уровня $\mathrm{HbA}_{1 \mathrm{c}}$ [6]. Заметное число детей и подростков прекращают использование помповой инсулинотерапии спустя несколько лет после перевода на НПИИ по разным причинам [6, 7].

Таким образом, выбор лучших кандидатов для НПИИ, у которых ожидается продолжительное использование помповой инсулинотерапии с положительным результатом, представляет собой серьезную клиническую проблему. Лишь в нескольких исследованиях были изучены отдельные факторы, которые могут быть использованы для оценки прогнозирования эффективности использования НПИИ [6-8].

\section{ЦЕЛЬ}

Оценить уровень гликемического контроля и факторы, влияющие на эффективность длительного применения НПИИ у детей.

\section{МЕТОДЫ}

\section{Дизайн исследования}

Объектом исследования являлась база данных специализированного регистра пациентов с СД1, переведенных на помповую инсулинотерапию. Проведен ретроспективный анализ.

\section{Критерии соответствия}

Критерии включения в анализ:

1. возраст от 1 до 18 лет на момент перевода на НПИИ;

2. длительность использования НПИИ не менее 3 лет до момента исследования;

3. наличие актуальных данных (анализируемых показателей) в регистре.

Критерии исключения из анализа:

1. СД не 1 типа, подтвержденный на момент исследования.

\section{Условия проведения}

Исследование проведено на базе детского отделения сахарного диабета ФГБУ «НМИЦ эндокринологии» Минздрава России. В соответствии с поставленной целью для последующего анализа были получены данные из специализированного регистра помповой инсулинотерапии Института детской эндокринологии ФГБУ «НМИЦ эндокринологии» Минздрава России (далее регистр).

Всего на момент исследования в регистре содержались данные 1471 пациента, из них 458 полностью отвечали критериям включения (рис. 1).

\section{Продолжительность исследования}

Ретроспективный анализ базы данных специализированного регистра пациентов с СД1, использующих помповую инсулинотерапию не менее 3 лет.

\section{Основной исход исследования}

Уровень $\mathrm{HbA}_{1 с}$ и доля (\%) участников с $\mathrm{HbA}_{1 c}<7,5 \%$ на момент исследования по сравнению с уровнем до перевода на НПИИ.

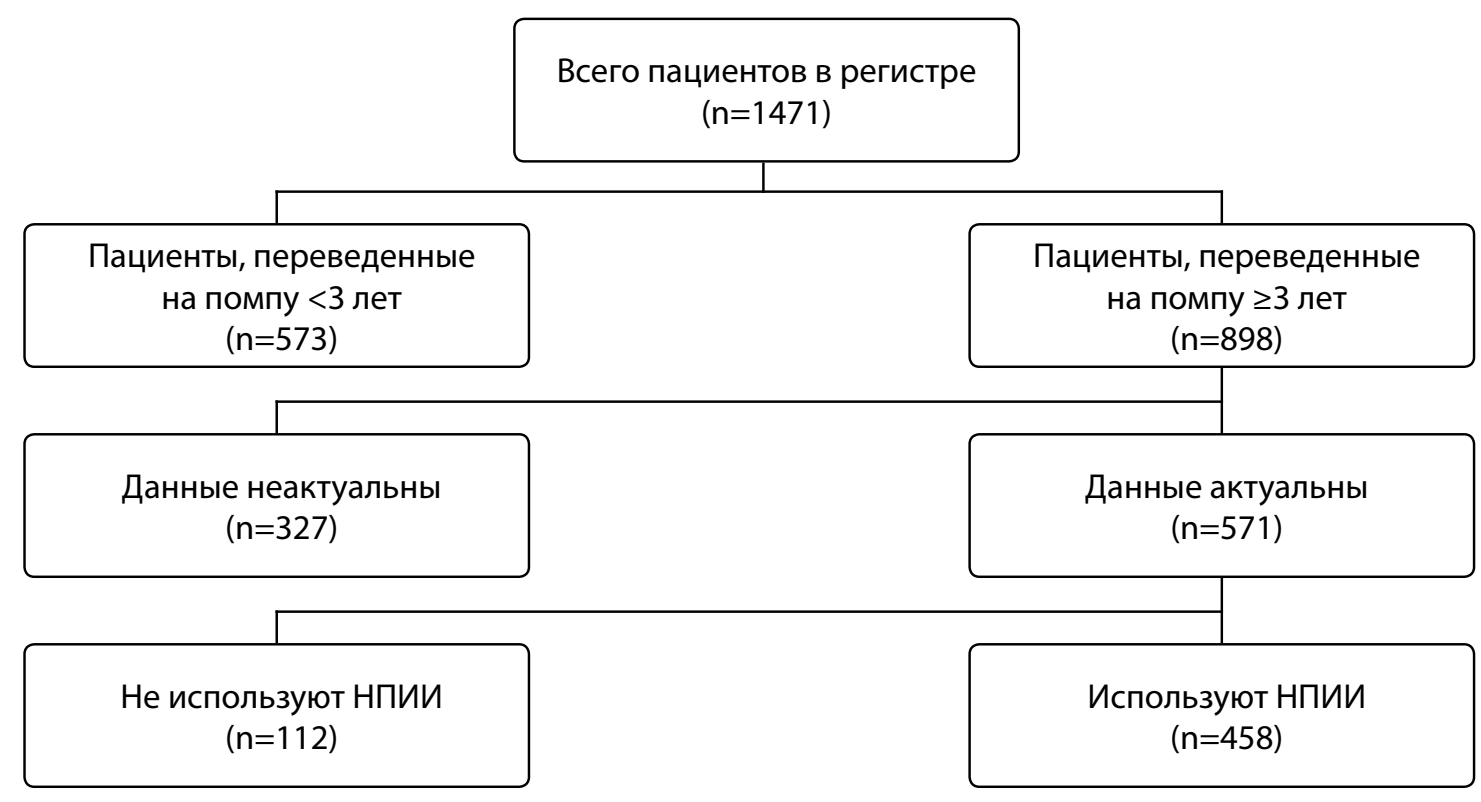

Рисунок 1. Блок-схема отбора участников исследования. 
Дополнительные исходы исследования

- Факторы, значимо ассоциированные с ответом на НПИИ.

- Частота, структура и факторы, ассоциированные с прекращением использования НПИИ.

\section{Методология исследования}

Данные в регистр вносились из медицинской документации пациентов при получении ими медицинской помощи в амбулаторных и стационарных условиях. Учитывались данные как лабораторных исследований, так и анамнеза.

\section{Анализируемые показатели}

Показатели возраста, длительности НПИИ, длительности СД1 представлены на дату последнего внесения актуальной информации в регистр.

При проведении анализа в качестве критерия ответа (положительного эффекта) на помповую инсулинотерапию было принято достижение уровня $\mathrm{HbA}_{1 c}$ на момент проведения исследования менее 7,5\% и/или снижение уровня $\mathrm{HbA}_{1 с}$ на $1 \%$ на момент проведения исследования по сравнению с исходным (до НПИИ) уровнем.

Использованием дополнительных настроек базального профиля считалось применение пациентом и/или его родителями временной базальной скорости и дополнительных базальных профилей. Использованием дополнительных болюсов считалось применение пациентом растянутого/квадратного, и/или двойного/ комбинированного, и/или суперболюса. Использованием непрерывного мониторинга глюкозы (НМГ) считалось применение пациентом системы НМГ в реальном времени или флеш-мониторинга не менее 60\% времени в течение предшествующих исследованию 3 мес. Бесплатным обеспечением расходными материалами к помпе считалось предоставление пациенту расходных материалов по месту жительства за счет бюджетных средств.

Данные о частоте диабетического кетоацидоза (ДКА) и тяжелой гипогликемии анализировались за весь период использования помповой инсулинотерапии.

\section{Анализ в подгруппах}

Помимо анализа в общей группе пациентов, показатели также анализировались в трех возрастных подгруппах в зависимости от возраста пациентов на момент перевода на НПИИ: от 0 до 6 лет, от 6 до 12 лет и от 12 до 18 лет.
Анализ эффективности НПИИ проводился в группе пациентов, продолжающих использовать помповую инсулинотерапию на момент проведения исследования. У пациентов, не использующих НПИИ на момент проведения исследования, проводился только анализ причин и факторов, ассоциированных с прекращением помповой инсулинотерапии.

\section{Этическая экспертиза}

Перед включением в регистр у пациентов или их законных представителей было получено информированное согласие на обработку и использование данных. Протокол исследования одобрен локальным Комитетом по этике ФГБУ «НМИЦ эндокринологии» Минздрава России (выписка из протокола №11 от 04.10.2015).

\section{Статистический анализ}

Размер выборки предварительно не рассчитывался. Обработка и анализ статистических данных проводились в программах Statistica 8.0 (StatSoft, США), SPSS Statistics v. 22 (IBM, США), MS Excel 2010 (Microsoft, США). Количественные данные представлены в виде медианы и интерквартильного размаха - Me (Q1-Q3) или среднего значения и 95\% доверительного интервала (ДИ) - М [95\% ДИ], качественные - в виде абсолютных значений (n) и/или частот (\%), данные о ДКА, тяжелой гипогликемии и частоте прекращения помповой терапии представлены в виде частоты эпизодов в пересчете на 100 пациентов в год. Различие между количественными признаками оценивалось с помощью U-критерия Манна-Уитни или критерия Уилкоксона для связных выборок с поправкой Бонферрони в случае множественных сравнений. Различия между качественными признаками оценивались с помощью критерия $\mathrm{X}^{2}$ (Хи-квадрат) или двустороннего точного критерия Фишера. Различия между частотами ДКА и тяжелой гипогликемии оценивались с помощью двухстороннего Z-критерия. Для выявления предикторов ответа на помповую терапию и причин отказа от помповой терапии использована логистическая регрессия, данные регрессионного анализа представлены в виде регрессионных коэффициентов (В), отношения шансов (ОШ) и 95\% ДИ. Значение р менее 0,05 считалось статистически значимым.

\section{РЕЗУЛЬТАТЫ}

Участники исследования

Клиническая характеристика пациентов, включенных в исследование, представлена в табл. 1.

Таблица 1. Характеристика пациентов, включенных в исследование

\begin{tabular}{lcc}
\hline \multicolumn{1}{c}{ Показатель } & Продолжают НПИИ & Прервали НПИИ \\
$\mathbf{n = 4 5 8}$ & 412 \\
\hline Пол, муж. & $47 \%$ & $48 \%$ \\
Возраст, годы & $14,2(10,8-17,8)$ & $16,2(12,6-19,5)$ \\
Длительность СД1, годы & $7,7(5,8-10,6)$ & $8,8(6,6-11,5)$ \\
Длительность помповой терапии, годы & $5(3,8-6,5)$ & $3(1,1-4,4)$ \\
НьА $_{1 c^{\prime}} \%$ & $8(7-9)$ & $8,2(7,1-9,4)$
\end{tabular}

Примечание. Данные представлены в виде Me (Q1-Q3)/\%. 
Основные результаты исследования

Гликированный гемоглобин ( $\left.\mathrm{Hb}_{1}\right)$

Средний уровень $\mathrm{HbA}_{1 с}$ во всей группе пациентов ( $n=458)$ статистически значимо снизился с 8,8\% на момент перевода на НПИИ до 8,1\% на момент проведения исследования (рис. 2). Таким образом, средняя разница между исходным и текущим уровнями составила 0,7\% [95\% ДИ 0,5-0,9]. Схожая тенденция в виде статистически значимого снижения уровня $\mathrm{HbA}_{1 с}$ на момент исследования относительно исходных значений наблюдалась при анализе пациентов во всех возрастных подгруппах. Так, средняя разница между исходным и текущим уровнями $\mathrm{HbA}_{1 с}$ составила: у пациентов в возрасте <6 лет $(\mathrm{n}=127)-0,9 \%$ [95\% ДИ 0,4-1,4]; у пациентов в возрасте 6-12 лет $(n=211)-0,5 \%$
[95\% ДИ 0,2-0,8]; у пациентов в возрасте 12-18 лет $(n=120)-0,8 \%[95 \%$ ДИ 0,5-1,1].

Число пациентов с уровнем $\mathrm{HbA}_{1 c}<7,5 \%$ в общей группе увеличилось более чем в 2 раза, с 17 до 36\%. Число пациентов с уровнем $\mathrm{HbA}_{1 c}<7,5 \%$ у пациентов в возрасте $<6$ лет ( $n=127)$ увеличилось с 20 до 50\%, у пациентов в возрасте 6-12 лет (n=211) - с 19 до 34\%, у пациентов в возрасте 12-18 лет $(\mathrm{n}=120)$ - с 11 до $26 \%$.

Изменение $\mathrm{HbA}_{1 c}$ после перевода на НПИИ зависело в значительной степени от исходного уровня $\mathrm{HbA}_{1 c}$. Так, динамика $\mathrm{HbA}_{1 с}$ на момент исследования по сравнению с исходным уровнем была следующей: у пациентов с $\mathrm{HbA}_{1 \mathrm{c}} \geq 9 \%$ - снижение на 2,0\% [95\% ДИ 1,7-2,4]; у пациентов с HbA 1 7,5-9\% - снижение на 0,2\% [95\% ДИ 0,0-0,3]; у пациентов с $\mathrm{HbA}_{1 c}<7,5 \%$ - увеличение на 0,8\% [95\% ДИ 0,5-1,1].
Возраст 0-18 лет $(\mathrm{n}=458)$

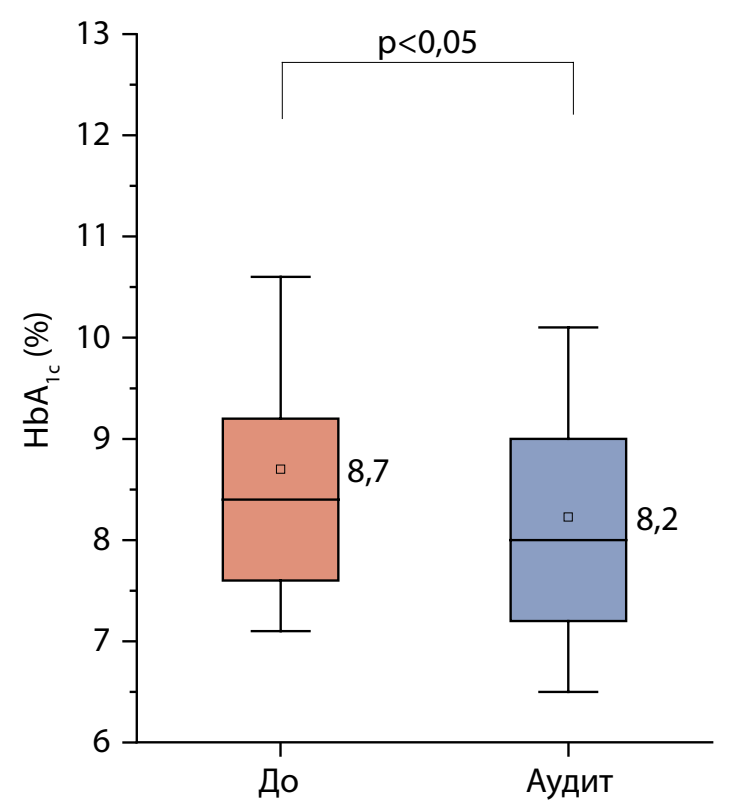

Возраст 6-12 лет $(\mathrm{n}=211)$

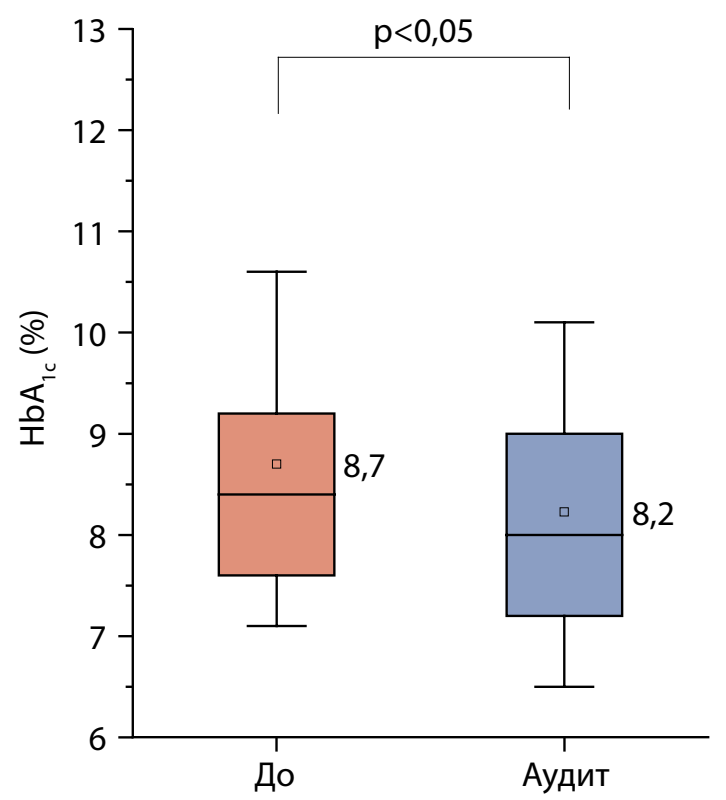

Возраст <6 лет $(\mathrm{n}=127)$

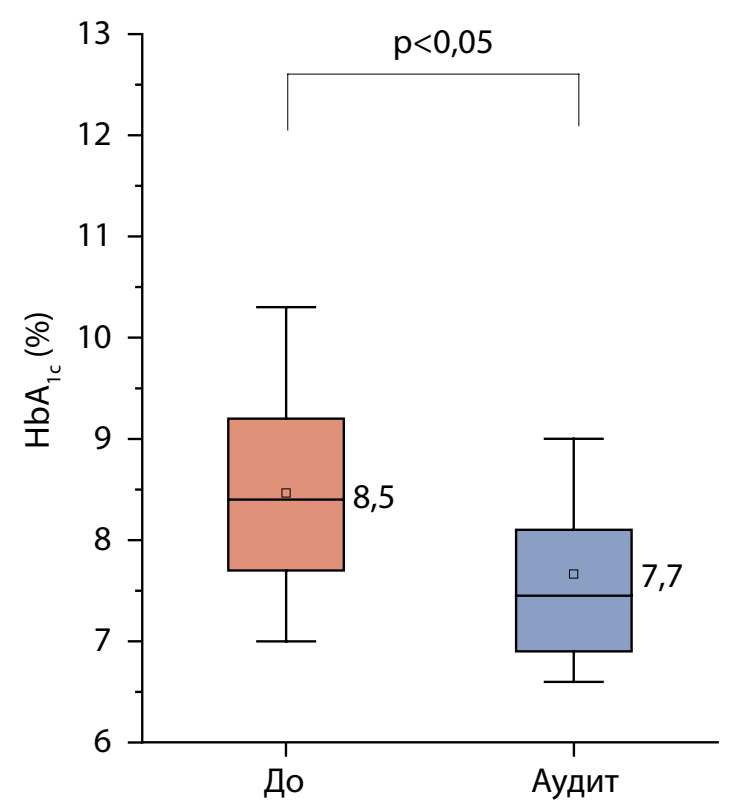

Возраст 12-18 лет $(n=120)$

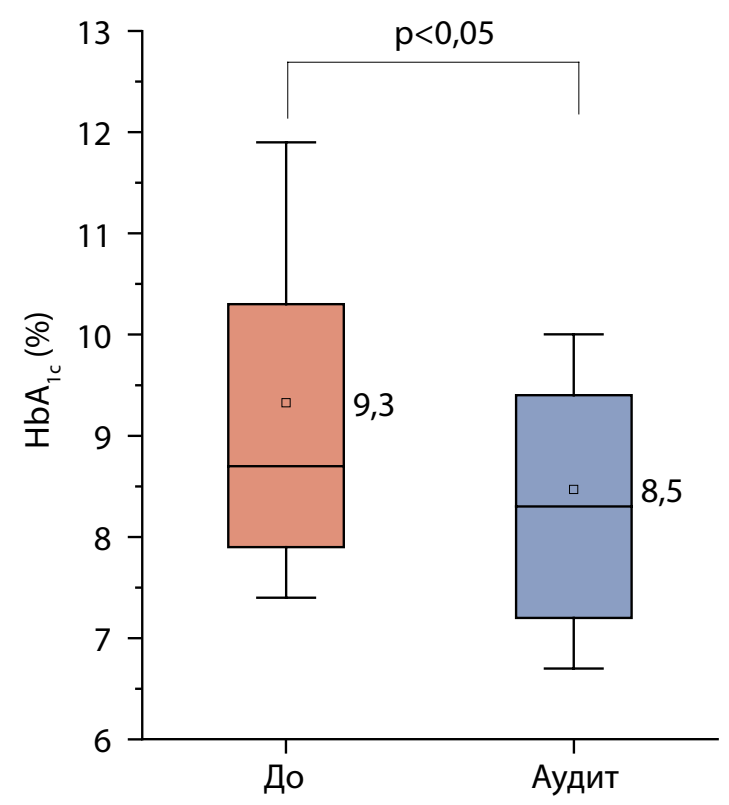

Рисунок 2. Изменение уровня гликированного гемоглобина на момент исследования (непрерывная подкожная инфузия инсулина) относительно исходного уровня (исходно) у пациентов в разных возрастных группах. Данные представлены в виде: M/Mе/Q1-Q3/10-90 перцентиль. P<0,05 статистически значимые различия по сравнению с уровнем гликированного гемоглобина до перевода на помпу. 
Возраст 0-18 лет $(\mathrm{n}=458)$

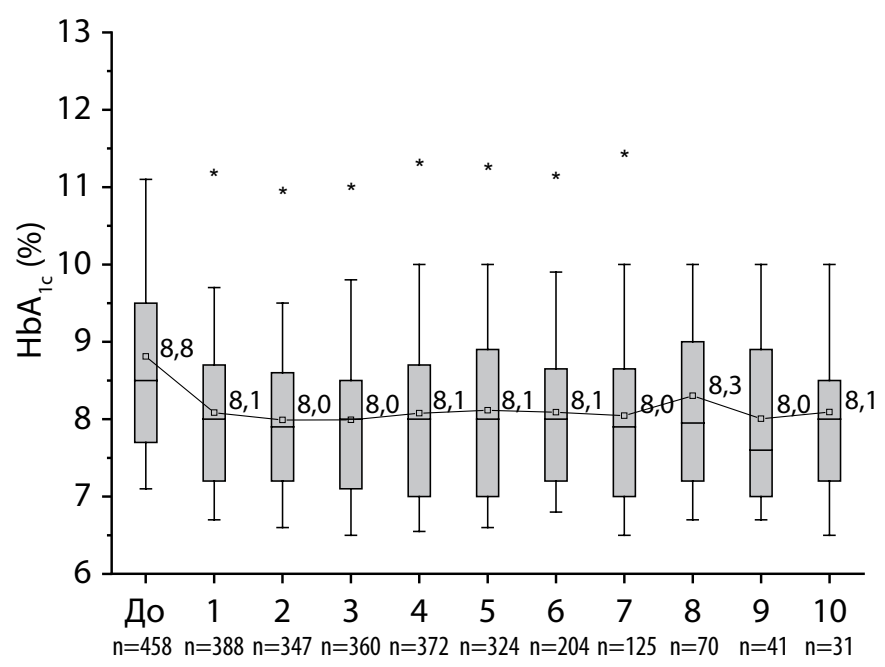

Годы помповой терапии (кол-во пациентов)
Возраст <6 лет $(\mathrm{n}=127)$

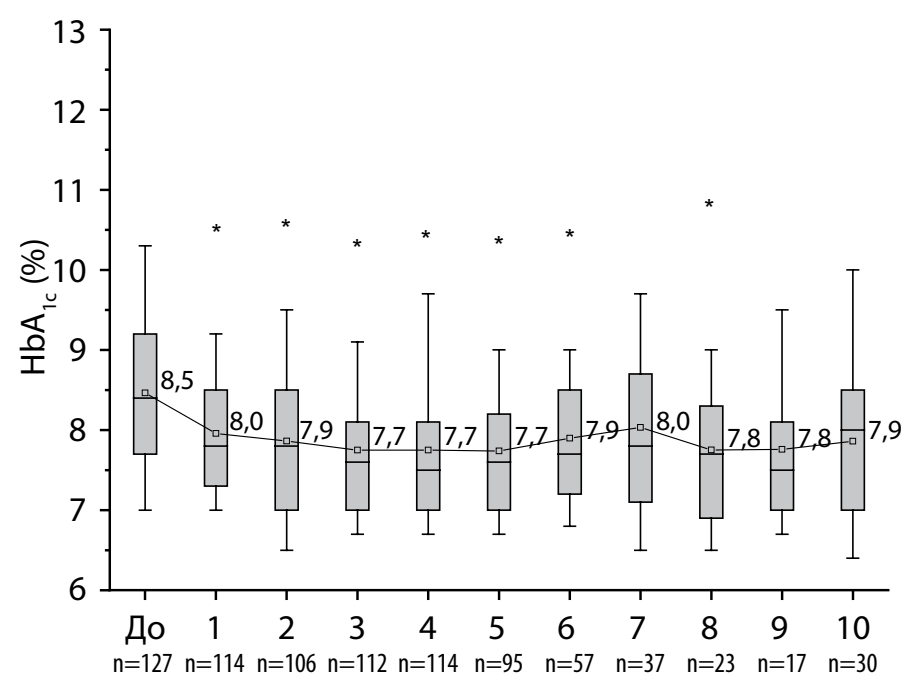

Годы помповой терапии (кол-во пациентов)

Возраст 12-18 лет $(\mathrm{n}=120)$

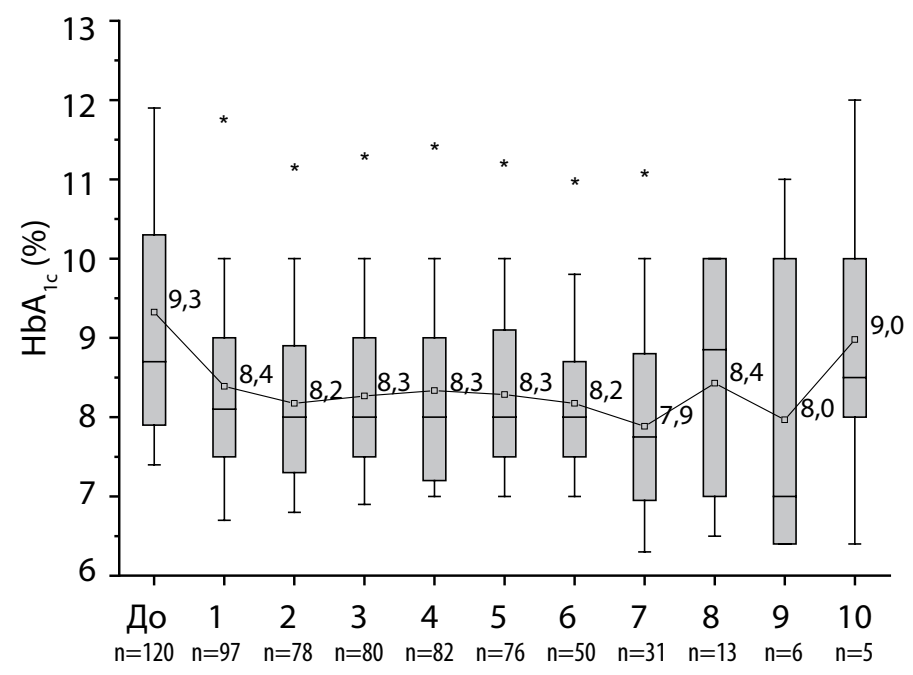

Годы помповой терапии (кол-во пациентов)

Рисунок 3. Изменение уровня гликированного гемоглобина в зависимости от длительности помповой терапии у пациентов в разных возрастных группах. Данные представлены в виде: M/Me/Q1-Q3/10-90 перцентиль. * - статистически значимые различия по сравнению с уровнем гликированного гемоглобина до перевода на помпу.

Динамика $\mathrm{HbA}_{1 с}$ за время использования инсулиновой помпы во всей группе пациентов и в разных возрастных подгруппах представлена на рис. 3. В общей группе пациентов $(n=458)$ в течение 7 лет после перевода на НПИИ показатели $\mathrm{HbA}_{1 с}$ были статистически значимо ниже исходного (перед НПИИ) уровня. В схожей манере показатели $\mathrm{HbA}_{1 с}$ были статистически значимо ниже: у пациентов в возрасте <6 лет $(n=127)$ в первые 6 лет и на 8-м году НПИИ; у пациентов в возрасте 6-12 лет $(\mathrm{n}=211)$ - только в первые 4 года НПИИ; у пациентов в возрасте $12-18$ лет $(n=120)$ показатели $\mathrm{HbA}_{1 с}$ были статистически значимо ниже в первые 7 лет НПИИ.

Частота компенсации углеводного обмена, определяемая по уровню $\mathrm{HbA}_{1 с}$ менее 7,0 и 7,5\% для всех пациентов, составила $36 \%$ и $18 \%$ соответственно. При этом число пациентов с уровнем $\mathrm{HbA}_{1 с}$ менее 7,0 и 7,5\% статистически значимо $(p<0,05)$, по сравнению с другими возрастными подгруппами, было больше в подгруппе пациентов, переведенных на помповую инсулинотерапию в возрасте <6 лет, и составило соответственно $50 \%$ и 25\% против 34\% и 18\% у пациентов в возрасте 6-12 лет; $26 \%$ и $12 \%$ у пациентов в возрасте $12-18$ лет.

\section{Дополнительные результаты исследования Ответ на помповую инсулинотерапию}

Среди всех пациентов 53\% (243) ответили, согласно принятым критериям (HbA на 1\%), на терапию НПИИ. По сравнению с пациентами, не ответившими на НПИИ на момент исследования, они были младше и имели более низкий исходный уровень $\mathrm{HbA}_{1 \mathrm{c}}$ (табл. 2).

Пациенты, ответившие на помповую терапию, на момент перевода на НПИИ были младше, имели более высокий уровень $\mathrm{HbA}_{1 c^{\prime}}$ при этом длительность СД1 значимо не различалась по сравнению с пациентами, 
Таблица 2. Клиническая характеристика и показатели, ассоциированные с ответом на помповую инсулинотерапию

\begin{tabular}{|c|c|c|c|c|c|}
\hline & \multirow{2}{*}{$\begin{array}{l}\text { Не ответили } \\
\mathrm{n}=215 \text { (47\%) }\end{array}$} & \multirow{2}{*}{$\begin{array}{c}\text { Положительный } \\
\text { эффект на НПИИ } \\
\text { n=243 (53\%) }\end{array}$} & \multirow{2}{*}{$\mathbf{p}$} & \multicolumn{2}{|c|}{ Логистическая регрессия } \\
\hline & & & & одномерная & многомерная \\
\hline Пол, муж. & $47 \%$ & $48 \%$ & $H / 3$ & & \\
\hline \multicolumn{6}{|c|}{ На момент исследования } \\
\hline Возраст, годы & $15,2(11,7-18,2)$ & $13,8(9,9-17,6)$ & $<0,05$ & & \\
\hline Длительность СД, годы & $8,3(6,2-10,6)$ & $7,6(5,6-10,6)$ & $H / 3$ & & \\
\hline $\begin{array}{l}\text { Длительность помповой терапии, } \\
\text { годы }\end{array}$ & $5,2(4,1-6,5)$ & $4,9(3,8-6,5)$ & $\mathrm{H} / 3$ & & \\
\hline $\mathrm{HbA}_{1 \mathrm{c}^{\prime}} \%$ & $8,6(8-10)$ & $7,1(6,7-7,6)$ & $<0,05$ & & \\
\hline Средняя частота самоконтроля & $5(4-7)$ & $5(4-8)$ & $\mathrm{H} / 3$ & & ** \\
\hline ДКА ${ }^{* * *}$ & 8,1 & 4,8 & $<0,05$ & * & \\
\hline Тяжелые гипогликемии*** & 2,8 & 2,3 & $\mathrm{H} / 3$ & & \\
\hline $\begin{array}{l}\text { Обеспечение расходными } \\
\text { материалами }\end{array}$ & $74 \%$ & $73 \%$ & $\mathrm{H} / 3$ & & \\
\hline $\begin{array}{l}\text { Использование дополнительных } \\
\text { базальных функций }\end{array}$ & $60 \%$ & $77 \%$ & $<0,05$ & * & $* *$ \\
\hline $\begin{array}{l}\text { Использование дополнительных } \\
\text { болюсов }\end{array}$ & $50 \%$ & $64 \%$ & $<0,05$ & * & \\
\hline Использование НМГ & $25 \%$ & $45 \%$ & $<0,05$ & * & ** \\
\hline \multicolumn{6}{|c|}{ На момент перевода на НПИИ } \\
\hline Возраст, годы & $9,6(6,5-12,5)$ & $8,2(4,6-11,9)$ & $<0,05$ & $*$ & \\
\hline Возраст <6 лет & $20 \%$ & $35 \%$ & $<0,05$ & * & \\
\hline Возраст 6-12 лет & $52 \%$ & $41 \%$ & $<0,05$ & * & \\
\hline Возраст 12-18 лет & $28 \%$ & $24 \%$ & $H / 3$ & & \\
\hline Длительность СД, годы & $2,4(1,3-4,5)$ & $2,2(1-4,2)$ & $\mathrm{H} / 3$ & & \\
\hline $\mathrm{HbA}_{1 \mathrm{c}^{\prime}} \%$ & $8,3(7,8-8,8)$ & $8,7(7,7-10,3)$ & $<0,05$ & * & $* *$ \\
\hline $\mathrm{HbA}_{1 \mathrm{c}}<7,5 \%$ & $16 \%$ & $18 \%$ & $\mathrm{H} / 3$ & & \\
\hline $\mathrm{HbA}_{1 \mathrm{c}} \geq 9 \%$ & $19 \%$ & $46 \%$ & $<0,05$ & * & \\
\hline
\end{tabular}

Примечание. Данные представлены в виде Ме (Q1-Q3)/\%; * и ** - уровень статистической значимости <0,05 для одномерной и многомерной логистической регрессии соответственно; *** — эпизодов на 100 пациентов в год или наличие/отсутствие для логистической регрессии.

не ответившими на НПИИ. Среди пациентов, ответивших на НПИИ, преобладали дети в возрасте менее 6 лет с уровнем $\mathrm{HbA}_{1 c}$ более 9\%, использовавшие дополнительные базальные и болюсные функции инсулиновой помпы и регулярно использовавшие НМГ. Из 147 пациентов, исходно имевших $\mathrm{HbA}_{1 с}$ более 9\%, на помповую инсулинотерапию ответили 107 (73\%), при этом уровень $\mathrm{HbA}_{1 с}$ снизился у 120 (82\%) из них.

По данным одномерного логистического регрессионного анализа, ответ на помповую инсулинотерапию был статистически значимо связан с меньшим возрастом в целом (ОШ 0,95 [95\% ДИ 0,91-0,99]; $\mathrm{p}=0,02)$ и в частности с возрастом до 6 лет до перевода на НПИИ (ОШ 2,2 [95\% ДИ 1,4-3,4]; $p<0,001$ ), более высоким уровнем $\mathrm{HbA}_{1 с}$ до перевода на НПИИ (ОШ 1,3 [95\% дИ 1,2-1,5]; $\mathrm{p}<0,001)$, уровнем $\mathrm{HbA}_{1 \mathrm{c}} \geq 9 \%$ до перевода на НПИИ (ОШ 3,7 [95\% ДИ 2,4-5,6]; $p<0,001$ ), использованием дополнительных базальных (ОШ 2,1 [95\% ДИ 1,4-3,1]; p<0,001) и болюсных (ОШ 1,7 [95\% ДИ $1,2-2,5] ; p=0,01)$ функций инсулиновой помпы, регу- лярным использованием НМГ (ОШ 2,5 [95\% ДИ 1,7-3,8]; $\mathrm{p}<0,001)$. По данным многомерного логистического регрессионного анализа, ответ на помповую инсулинотерапию был статистически значимо связан с более

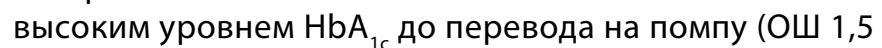
[95\% ДИ 1,3-1,7]; $\mathrm{p}<0,001)$, большей частотой самоконтроля (ОШ 1,1 [95\% ДИ 1-1,2]; $\mathrm{p=0,01),} \mathrm{использовани-}$ ем дополнительных базальных функций инсулиновой помпы (ОШ 1,8 [95\% ДИ 0,9-3,7]; $p=0,04)$ и регулярным использованием НМГ (ОШ 3,2 [95\% дИ 1,9-5,4]; $\mathrm{p}<0,001)$.

Анализ причин и факторов, ассоциированных с прекращением помповой инсулинотерапии у детей с СД1

Анализ структуры причин и факторов, ассоциированных с прекращением НПИИ, проводился у 112 пациентов, не вошедших в основной анализ, которые на момент проведения исследования не использовали инсулиновую помпу. Сравнительная характеристика пациентов, прекративших и продолжающих использование НПИИ, представлена в табл. 3. 
Таблица 3. Показатели, ассоциированные с прекращением использования помповой инсулинотерапии

\begin{tabular}{|c|c|c|c|c|c|}
\hline \multirow{2}{*}{ Показатели } & \multirow{2}{*}{$\begin{array}{l}\text { Не используют } \\
n=112\end{array}$} & \multirow{2}{*}{$\begin{array}{c}\text { Используют } \\
n=458\end{array}$} & \multirow{2}{*}{$\mathbf{p}$} & \multicolumn{2}{|c|}{ Логистическая регрессия } \\
\hline & & & & одномерная & многомерная \\
\hline Пол, муж. & $48 \%$ & $47 \%$ & $\mathrm{H} / 3$ & & \\
\hline Возраст (до помпы), годы & $10,4(6,8-13,3)$ & $8,9(5,7-12,2)$ & $<0,05$ & * & ** \\
\hline Возраст <6 лет (до помпы) & 21 & 28 & $\mathrm{H} / 3$ & & \\
\hline Возраст 6-12 лет (до помпы) & 39 & 46 & $\mathrm{H} / 3$ & & \\
\hline Возраст 12-18 лет (до помпы) & 40 & 26 & $<0,05$ & * & \\
\hline Длительность СД (до помпы), годы & $2,4(1,1-5,3)$ & $2,2(1,2-4,4)$ & $H / 3$ & & \\
\hline $\mathrm{HbA}_{1 с}$ (до помпы), \% & $8,5(7,6-9,7)$ & $8,5(7,7-9,5)$ & $\mathrm{H} / 3$ & & \\
\hline $\mathrm{HbA}_{1 с}<7,5 \%$ (до помпы) & $21 \%$ & $17 \%$ & $\mathrm{H} / 3$ & & \\
\hline $\mathrm{HbA}_{1 c} \geq 9 \%$ (до помпы) & $40 \%$ & $33 \%$ & $\mathrm{H} / 3$ & & \\
\hline Частота самоконтроля & $5(4-8)$ & $5(4-8)$ & $\mathrm{H} / 3$ & & \\
\hline ДКА за время помповой терапии*** & 8,5 & 6,4 & $\mathrm{H} / 3$ & & \\
\hline $\begin{array}{l}\text { Тяжелые гипогликемии за время } \\
\text { помповой терапии*** }\end{array}$ & 4,5 & 2,5 & $<0,05$ & & \\
\hline Использование НМГ & $28 \%$ & $38 \%$ & $<0,05$ & * & \\
\hline
\end{tabular}

Примечание. Данные представлены в виде Ме (Q1-Q3)/\%; $и$ ** - уровень статистической значимости <0,05 для одномерной и многомерной логистической регрессии соответственно; *** - эпизодов на 100 пациентов в год или наличие/отсутствие для логистической регрессии.

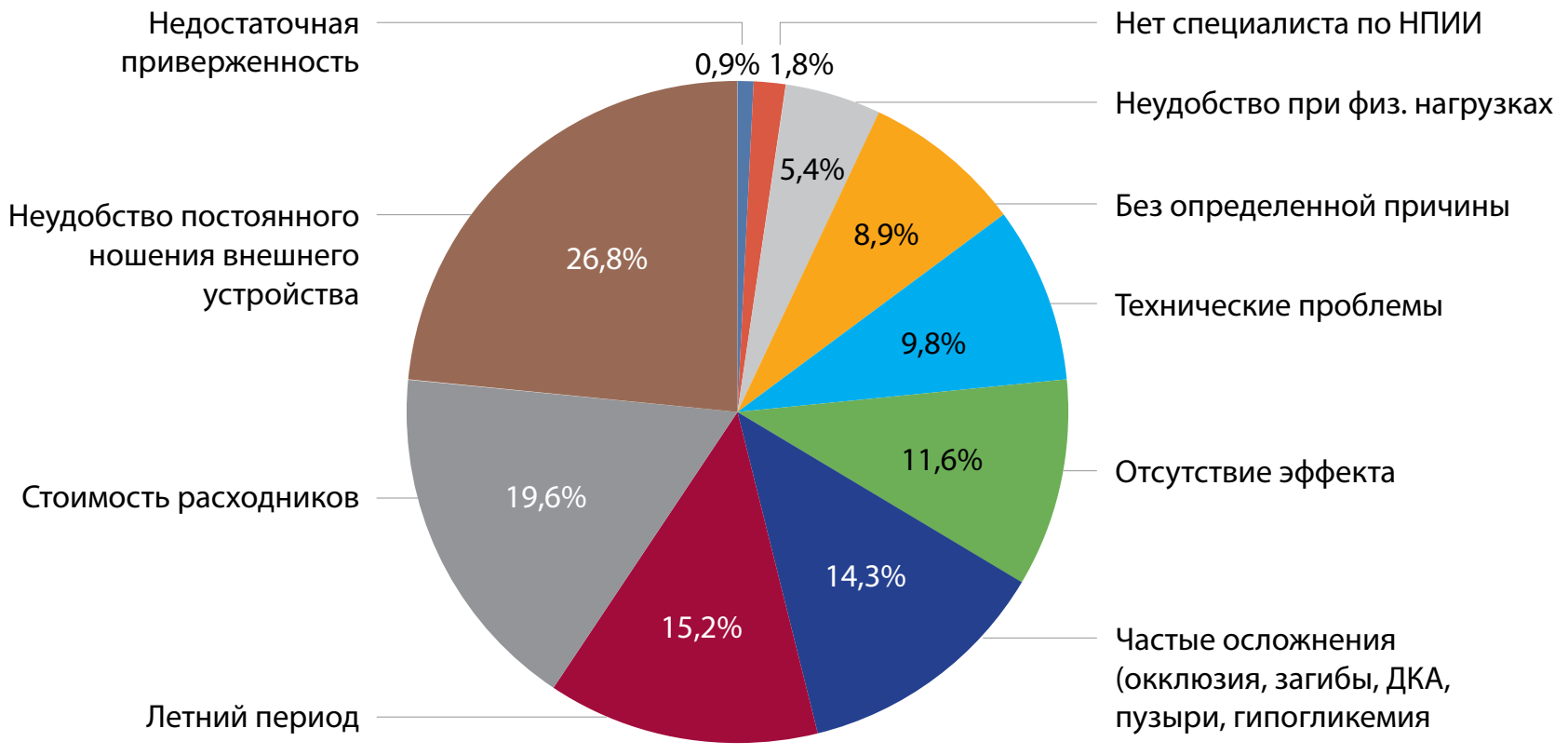

Рисунок 4. Структура причин и частота прекращения использования помповой инсулинотерапии.

Из 112 пациентов, прекративших использование НПИИ, 18 человек (16,1\%) сняли инсулиновую помпу временно, на летнее время, и в дальнейшем планировали вернуться к ней. Таким образом, исходя из числа прекративших и продолжавших использовать помповую инсулинотерапию, общая частота отказа от НПИИ составила 19,6\% (16,5\% без учета снявших помпу на время). С учетом длительности поповой терапии до момента ее прекращения, частота прекращения НПИИ составила 3,9 случая на 100 пациентов в год (3,4 случая на 100 пациентов в год без учета снявших помпу на время). Чаще всего пациенты прекращали использовать НПИИ в возрасте от 12 до 18 лет. На момент проведения исследова- ния пациенты, прекратившие использование инсулиновой помпы, были старше и имели более высокий уровень $\mathrm{HbA}_{1 c}$ по сравнению с пациентами, продолжающими использование НПИИ.

Структура причин, по которым пациенты прекратили использовать инсулиновую помпу, представлена на рис. 4. Основными причинами прекращения использования инсулиновой помпы в 47,4\% случаев являлось неудобство ее использования (неудобство постоянного ношения внешнего устройства - 26,8\%, неудобство в летнее время - 15,2\% и при физических нагрузках - 5,4\%). Другими причинами прекращения использования инсулиновой помпы были: стоимость 
расходных материалов (нет возможности приобретать самостоятельно, не получали бесплатно) - 19,6\%; нежелательные явления при использовании помпы, такие как частые закупорки инфузионного набора, загибы катетера, эпизоды ДКА, гипогликемии - 14,3\%; отсутствие эффекта - 11,6\%; технические проблемы - 9,8\%; отсутствие специалиста по НПИИ по месту жительства; недостаточная приверженность ребенка к лечению - 0,9\%. Кроме того, в 8,9\% случаев пациенты не указали определенной причины прекращения использования НПИИ.

Пациенты, прекратившие использование помповой терапии, на момент перевода на НПИИ были старше, и у них была выше частота тяжелой гипогликемии за время использования помпы (табл. 3). Среди пациентов, прекративших использование помповой терапии, преобладали дети в возрасте 12-18 лет, не использующие на регулярной основе НМГ.

По данным одномерного логистического регрессионного анализа, прекращение помповой терапии было статистически значимо связано с большим возрастом в целом (ОШ 1,1 [95\% ДИ 1-1,1]; $p=0,02$ ) и в частности с возрастом 12-18 лет на момент перевода на НПИИ (ОШ 1,9 [95\% ДИ 1,2-2,9]; p<0,01). В то же время регулярное использование НМГ было связано с меньшей вероятностью прекращения использования НПИИ (ОШ 0,6 [95\% ДИ 0,4-1]; $p=0,046$ ) (приложение 3).

По данным многомерного логистического регрессионного анализа, прекращение помповой терапии было статистически значимо связано только с большим возрастом на момент перевода на НПИИ (ОШ 1,1 [95\% ДИ 1-1,1]; $\mathrm{p}=0,04)$ (приложение 4).

\section{ОБСУЖДЕНИЕ}

По результатам проведенного исследования было показано, что НПИИ является эффективным методом инсулинотерапии, который позволяет достигнуть более низкого уровня $\mathrm{HbA}_{1 с}$ по сравнению с исходными значениями $(-0,7 \%)$, что сопровождается увеличением числа пациентов, достигших целевого уровня $\mathrm{HbA}_{1 c}(<7,5 \%)$, с 17 до 36\%. После инициации НПИИ отмечается в целом длительная положительная динамика в течение 7 лет, при этом большая длительность положительного эффекта от перевода на помповую инсулинотерапию отмечается у пациентов, которые были переведены в возрасте 0-6 и 12-18 лет. Данные результаты во многом согласуются с результатами исследования, проведенного в 2016 г. [6], за исключением несколько большей длительности эффекта от НПИИ, показанного по результатам настоящего исследования.

Лучший ответ на НПИИ отмечался у пациентов, переведенных на помповую инсулинотерапию в возрасте до 6 лет и с уровнем $\mathrm{HbA}_{1 с}$ более 9\%, а также использовавших дополнительные базальные и болюсные функции помпы, применявшиеся на регулярной основе НМГ. Данные результаты указывают, с одной стороны, на необходимость перевода на НПИИ в более раннем возрасте и, с другой стороны, подчеркивают тот факт, что сам по себе уровень $\mathrm{HbA}_{1 с}$ не должен являться основанием для отказа от перевода на помповую инсулинотерапию. Использование НМГ и больших возможностей инсулиновых помп являются хорошо известными факторами лучшего гликемического контроля [9-12], что указывает на необходимость обучения и мотивирования пациентов, родителей и врачей на использование этих возможностей.

При анализе причин и факторов, ассоциированных с прекращением использования помповой инсулинотерапии, показано, что число детей, отказавшихся от инсулиновых помп за все время с момента перевода на НПИИ, в исследованной выборке составило $20 \%$. В пересчете на длительность помповой терапии частота прекращения НПИИ составила 3,9 случая на 100 пациентов в год. В структуре причин прекращения использования помповой инсулинотерапии преобладало неудобство использования и ношения инсулиновой помпы (47,7\%). Факторами риска прекращения помповой инсулинотерапии были подростковый возраст (12-18 лет) на момент перехода на НПИИ и частые эпизоды тяжелой гипогликемии. В свою очередь, использование НМГ снижало риски отказа от помпы. Полученные результаты согласуются с ранее опубликованными данными регистра и отражают общемировые тенденции $[6,7]$. По сравнению с предыдущими результатами частота прекращения использования НПИИ стала меньше (3,9 против 4,6 случая на 100 пациентов в год), хотя структура причин не претерпела значительных изменений.

\section{ЗАКЛЮЧЕНИЕ}

У детей и подростков с СД1 перевод на НПИИ сопровождался снижением уровня $\mathrm{HbA}_{1 с}$ на 0,7\% и увеличением числа компенсированных пациентов с 17 до 36\%. Лучший ответ отмечался при переводе на помповую инсулинотерапию пациентов в возрасте до 6 лет, с НbA 6 более 9\%, а также у пациентов, регулярно использующих дополнительные болюсные и базальные функции и НМГ. Число детей, прекративших использование НПИИ, составило 20\%, в среднем через 5,6 года. Основной причиной прекращения использования инсулиновой помпы являлось неудобство использования и ношения - в 47,7\% случаев. Были выявлены факторы риска отказа от помпы: старший возраст перевода на НПИИ и частые эпизоды тяжелой гипогликемии.

\section{ДОПОЛНИТЕЛЬНАЯ ИНФОРМАЦИЯ}

Источник финансирования. Работа выполнена в рамках национальной благотворительной программы помощи детям с эндокринными заболеваниями «Альфа-Эндо» при финансовой поддержке фонда «КАФ».

Конфликт интересов. Авторы декларируют отсутствие явных и потенциальных конфликтов интересов, связанных с публикацией настоящей статьи.

Участие авторов. Петеркова В.А. - научное руководство, дизайн и планирование исследования; Лаптев Д.Н. - выгрузка данных из регистра, анализ и статистическая обработка полученных данных, написание текста; Емельянов А.О. - ведение регистра помповой инсулинотерапии, выгрузка данных из регистра, написание и редактирование текста; Медведева Е.Д. - ведение регистра помповой инсулинотерапии, выгрузка данных из регистра; Переверзева С.В. - ведение регистра помповой инсулинотерапии, выгрузка данных из регистра. 


\section{ДОПОЛНИТЕЛЬНЫЕ МАТЕРИАЛЫ}

Приложение 1. Результаты одномерной логистической регрессии. Данные представлены в виде регрессионных коэффициентов (В), отношения шансов (ОШ) и 95\% доверительного интервала (ДИ)

\begin{tabular}{|c|c|c|c|c|c|c|c|c|}
\hline Показатели & B & S.E. & Wald & df & $\mathbf{p}$ & ОШ & \multicolumn{2}{|c|}{ 95\% ди } \\
\hline Пол, муж. & 0,012 & 0,187 & 0,004 & 1 & 0,95 & 1,0 & 0,7 & 1,5 \\
\hline \multicolumn{9}{|c|}{ На момент исследования } \\
\hline Частота самоконтроля & 0,04 & 0,03 & 1,43 & 1,00 & 0,23 & 1,0 & 1,0 & 1,1 \\
\hline ДКА за время помповой терапии & $-0,652$ & 0,229 & 8,099 & 1 & $<0,01$ & 0,5 & 0,3 & 0,8 \\
\hline $\begin{array}{l}\text { Тяжелые гипогликемии за время } \\
\text { помповой терапии }\end{array}$ & $-0,289$ & 0,315 & 0,844 & 1 & 0,36 & 0,7 & 0,4 & 1,4 \\
\hline $\begin{array}{l}\text { Обеспечение расходными } \\
\text { материалами }\end{array}$ & 0 & 0,214 & 0,075 & 1 & 0,78 & 0,9 & 0,6 & 1,4 \\
\hline $\begin{array}{l}\text { Использование дополнительных } \\
\text { базальных функций }\end{array}$ & 0,719 & 0,206 & 12,147 & 1 & $<0,01$ & 2,1 & 1,4 & 3,1 \\
\hline $\begin{array}{l}\text { Использование дополнительных } \\
\text { болюсов }\end{array}$ & 0,533 & 0,192 & 7,667 & 1 & 0,01 & 1,7 & 1,2 & 2,5 \\
\hline Использование НМг & 1 & 0,204 & 20,512 & 1 & $<0,01$ & 2,5 & 1,7 & 3,8 \\
\hline \multicolumn{9}{|c|}{ На момент перевода на НПИИ } \\
\hline Возраст (до помпы), годы & $-0,05$ & 0,02 & 5,15 & 1,00 & 0,02 & 0,95 & 0,9 & 1,0 \\
\hline Возраст <6 лет (до помпы) & 0,796 & 0,218 & 13,277 & 1 & $<0,01$ & 2,2 & 1,4 & 3,4 \\
\hline Возраст 6-12 лет (до помпы) & $-0,458$ & 0,189 & 5,89 & 1 & 0,02 & 0,6 & 0,4 & 0,9 \\
\hline Возраст 12-18 лет (до помпы) & $-0,211$ & 0,213 & 0,986 & 1 & 0,32 & 0,8 & 0,5 & 1,2 \\
\hline Длительность СД (до помпы), годы & $-0,017$ & 0,031 & 0,298 & 1 & 0,59 & 1,0 & 0,9 & 1,0 \\
\hline $\mathrm{HbA}_{1 с}$ (до помпы), \% & 0,28 & 0,07 & 18,63 & 1,00 & $<0,01$ & 1,3 & 1,2 & 1,5 \\
\hline $\mathrm{HbA}_{1 c}<7,5 \%$ (до помпы) & 0,15 & 0,25 & 0,34 & 1,00 & 0,56 & 1,2 & 0,7 & 1,9 \\
\hline $\mathrm{HbA}_{1 c} \geq 9 \%$ (до помпы) & 1,30 & 0,22 & 35,21 & 1,00 & $<0,01$ & 3,7 & 2,4 & 5,6 \\
\hline
\end{tabular}

Приложение 2. Результаты многомерной логистической регрессии. Данные представлены в виде регрессионных коэффициентов (В), отношения шансов (ОШ) и 95\% доверительного интервала (ДИ)

\begin{tabular}{|c|c|c|c|c|c|c|c|c|}
\hline Показатели & B & S.E. & Wald & df & $\mathbf{p}$ & ОШ & \multicolumn{2}{|c|}{ 95\% ди } \\
\hline Пол, муж. & 0,11 & 0,21 & 0,26 & 1,00 & 0,61 & 1,1 & 0,7 & 1,7 \\
\hline \multicolumn{9}{|c|}{ На момент исследования } \\
\hline $\begin{array}{l}\text { Использование дополнительных } \\
\text { базальных функций }\end{array}$ & 0,61 & 0,35 & 3,02 & 1,00 & 0,04 & 1,8 & 0,9 & 3,7 \\
\hline $\begin{array}{l}\text { Использование дополнительных } \\
\text { болюсов }\end{array}$ & 0,09 & 0,33 & 0,08 & 1,00 & 0,77 & 1,1 & 0,6 & 2,1 \\
\hline Использование НМГ & 1,17 & 0,26 & 20,32 & 1,00 & $<0,01$ & 3,2 & 1,9 & 5,4 \\
\hline $\begin{array}{l}\text { Обеспечение расходными } \\
\text { материалами }\end{array}$ & $-0,10$ & 0,25 & 0,16 & 1,00 & 0,69 & 0,9 & 0,6 & 1,5 \\
\hline Частота самоконтроля & 0,09 & 0,04 & 6,22 & 1,00 & 0,01 & 1,1 & 1,0 & 1,2 \\
\hline \multicolumn{9}{|c|}{ На момент перевода на НПИИ } \\
\hline Возраст (до помпы), годы & $-0,01$ & 0,03 & 0,18 & 1,00 & 0,67 & 1,0 & 0,9 & 1,1 \\
\hline Длительность СД (до помпы), годы & 0,02 & 0,04 & 0,25 & 1,00 & 0,62 & 1,0 & 0,9 & 1,1 \\
\hline $\mathrm{HbA}_{1 с}$ (до помпы), \% & 0,40 & 0,08 & 27,14 & 1,00 & $<0,01$ & 1,5 & 1,3 & 1,7 \\
\hline Константа & $-4,75$ & 0,90 & 28,13 & 1,00 & $<0,01$ & 0,0 & & \\
\hline
\end{tabular}


Приложение 3. Результаты одномерной логистической регрессии. Данные представлены в виде регрессионных коэффициентов (В), отношения шансов (ОШ) и 95\% доверительного интервала (ДИ)

\begin{tabular}{|c|c|c|c|c|c|c|c|c|}
\hline Показатели & B & S.E. & Wald & df & $\mathbf{p}$ & Ош & \multicolumn{2}{|c|}{ 95\% ди } \\
\hline Пол, муж. & $-0,01$ & 0,21 & 0,00 & 1,00 & 0,96 & 1,0 & 0,7 & 1,5 \\
\hline Возраст (до помпы), годы & 0,06 & 0,03 & 5,97 & 1,00 & 0,02 & 1,1 & 1,0 & 1,1 \\
\hline Возраст <6 лет (до помпы) & $-0,40$ & 0,26 & 2,38 & 1,00 & 0,12 & 0,7 & 0,4 & 1,1 \\
\hline Возраст 6-12 лет (до помпы) & $-0,28$ & 0,22 & 1,67 & 1,00 & 0,20 & 0,8 & 0,5 & 1,2 \\
\hline Возраст 12-18 лет (до помпы) & 0,64 & 0,22 & 8,39 & 1,00 & $<0,01$ & 1,9 & 1,2 & 2,9 \\
\hline Длительность СД (до помпы), годы & 0,03 & 0,03 & 0,54 & 1,00 & 0,46 & 1,0 & 1,0 & 1,1 \\
\hline $\mathrm{HbA}_{1 с}$ (до помпы), \% & $-0,01$ & 0,06 & 0,03 & 1,00 & 0,87 & 1,0 & 0,9 & 1,1 \\
\hline $\mathrm{HbA}_{1 c}<7,5 \%$ (до помпы) & 0,26 & 0,27 & 0,93 & 1,00 & 0,34 & 1,3 & 0,8 & 2,2 \\
\hline $\mathrm{HbA}_{1 c} \geq 9 \%$ (до помпы) & 0,32 & 0,22 & 2,12 & 1,00 & 0,15 & 1,4 & 0,9 & 2,1 \\
\hline Частота самоконтроля & 0,00 & 0,04 & 0,00 & 1,00 & 0,95 & 1,0 & 0,9 & 1,1 \\
\hline ДКА за время помповой терапии & 0,13 & 0,48 & 0,07 & 1,00 & 0,79 & 1,1 & 0,4 & 2,9 \\
\hline $\begin{array}{l}\text { Тяжелые гипогликемии за время } \\
\text { помповой терапии }\end{array}$ & 1,27 & 0,92 & 1,91 & 1,00 & 0,17 & 3,6 & 0,6 & 21,5 \\
\hline Использование НМГ & $-0,47$ & 0,24 & 3,97 & 1,00 & 0,046 & 0,6 & 0,4 & 1,0 \\
\hline
\end{tabular}

Приложение 4. Результаты многомерной логистической регрессии. Данные представлены В виде, регрессионных коэффициентов (В), отношения шансов (ОШ) и 95\% доверительного интервала (ДИ)

\begin{tabular}{|c|c|c|c|c|c|c|c|c|}
\hline Показатели & B & S.E. & Wald & df & $\mathbf{p}$ & Ош & \multicolumn{2}{|c|}{ 95\% ди } \\
\hline Пол, муж. & $-0,03$ & 0,23 & 0,02 & 1,00 & 0,88 & 1,0 & 0,6 & 1,5 \\
\hline Возраст (до помпы), годы & 0,06 & 0,04 & 3,30 & 1,00 & 0,04 & 1,1 & 1,0 & 1,1 \\
\hline Длительность СД (до помпы), годы & $-0,07$ & 0,05 & 1,98 & 1,00 & 0,16 & 0,9 & 0,9 & 1,0 \\
\hline $\mathrm{HbA}_{1 с}$ (до помпы), \% & 0,01 & 0,07 & 0,01 & 1,00 & 0,94 & 1,0 & 0,9 & 1,1 \\
\hline Частота самоконтроля & 0,00 & 0,04 & 0,00 & 1,00 & 0,96 & 1,0 & 0,9 & 1,1 \\
\hline ДКА за время помповой терапии & 0,02 & 0,48 & 0,00 & 1,00 & 0,97 & 1,0 & 0,4 & 2,6 \\
\hline $\begin{array}{l}\text { Тяжелые гипогликемии за время } \\
\text { помповой терапии }\end{array}$ & 1,49 & 0,99 & 2,26 & 1,00 & 0,13 & 4,4 & 0,6 & 30,9 \\
\hline Использование НМГ & $-0,37$ & 0,29 & 1,62 & 1,00 & 0,20 & 0,7 & 0,4 & 1,2 \\
\hline Constant & $-1,90$ & 0,79 & 5,73 & 1,00 & 0,02 & 0,2 & & \\
\hline
\end{tabular}

\section{СПИСОК ЛИТЕРАТУРЫ | REFERENCES}

1. Zinman B, Genuth S, Nathan DM. The Diabetes Control and Complications Trial/Epidemiology of Diabetes Interventions and Complications Study: 30th Anniversary Presentations. Diabetes Care. 2014;37(1):8-8. doi: https://doi.org/10.2337/dc13-2111

2. Sherr JL, Tauschmann M, Battelino T, et al. ISPAD Clinical Practice Consensus Guidelines 2018: Diabetes technologies. Pediatr Diabetes. 2018;19:302-325. doi: https://doi.org/10.1111/pedi.12731

3. Дедов И.И. и др. Инсулиновая помпа (помощь врачу и пачиенту для эфффективного управления диабетом). - М.; 2014. 126 с. [Dedov II et al. Insulinovaya pompa (pomocsh vrachu i pacientu dlya effektivnogo upravlenia diabetom). Moscow; 2014. 126 p. (In Russ.)].

4. Benkhadra K, Alahdab F, Tamhane SU, et al. Continuous subcutaneous insulin infusion versus multiple daily injections in individuals with type 1 diabetes: a systematic review and meta-analysis. Endocrine. 2017;55(1):77-84. doi: https://doi.org/10.1007/s12020-016-1039-x

5. Pańkowska E, Błazik M, Dziechciarz P, et al. Continuous subcutaneous insulin infusion vs. multiple daily injections in children with type 1 diabetes: a systematic review and meta-analysis of randomized control trials. Pediatr Diabetes. 2009;10(1):52-58. doi: https://doi.org/10.1111/j.1399-5448.2008.00440.x

6. Лаптев Д.Н., Переверзева С.В., Емельянов А.О., Петеркова В.А. Мониторинг применения помповой инсулинотерапии у детей, подростков и молодых пациентов с сахарным диабетом 1 типа в Российской Федерации // Проблемы эндокринологии. 2018. — T. 64. — №2. — C. 85-92. [Laptev DN, Pereverzeva SV, Emelyanov AO, Peterkova VA. Monitoring of insulin pump therapy in children, adolescents, and young adults with type 1 diabetes mellitus in the Russian Federation. Probl Endocrinol. 2018;64(2):85-92. (In Russ.)] doi: https://doi.org/10.14341/probl8756

7. Tanenbaum ML, Hanes SJ, Miller KM, et al. Diabetes Device Use in Adults With Type 1 Diabetes: Barriers to Uptake and Potential Intervention Targets. Diabetes Care. 2017;40(2):181-187. doi: https://doi.org/10.2337/dc16-1536 
8. Overgaard Ingeholm I, Svensson J, Olsen B, et al. Characterization of metabolic responders on CSII treatment amongst children and adolescents in Denmark from 2007 to 2013. Diabetes Res Clin Pract. 2015;109(2):279-286. doi: https://doi.org/10.1016/j.diabres.2015.05.027

9. Pańkowska E, Szypowska A, Lipka M, et al. Application of novel dual wave meal bolus and its impact on glycated hemoglobin A1c level in children with type 1 diabetes. Pediatr Diabetes. 2009;10(5):298-303. doi: https://doi.org/10.1111/j.1399-5448.2008.00471.x

10. Ramotowska A, Golicki D, Dżygało K, Szypowska A. The Effect of Using the Insulin Pump Bolus Calculator Compared to Standard Insulin Dosage Calculations in Patients with Type 1 Diabetes Mellitus - Systematic Review. Exp Clin Endocrinol Diabetes. 2013;121(05):248-254. doi: https://doi.org/10.1055/s-0032-1331708
11. Pańkowska E, Błazik M, Groele L. Does the Fat-Protein Meal Increase Postprandial Glucose Level in Type 1 Diabetes Patients on Insulin Pump: The Conclusion of a Randomized Study. Diabetes Technol Ther. 2012;14(1):16-22. doi: https://doi.org/10.1089/dia.2011.0083

12. Langendam M, Luijf YM, Hooft L, et al. Continuous glucose monitoring systems for type 1 diabetes mellitus. Cochrane Database Syst Rev. 2012;19:75-83. doi: https://doi.org/10.1002/14651858.CD008101.pub2

13. Phelan H, Lange K, Cengiz E, et al. ISPAD Clinical Practice Consensus Guidelines 2018: Diabetes education in children and adolescents. Pediatr Diabetes. 2018;19:75-83. doi: https://doi.org/10.1111/pedi.12762

\section{ИНФОРМАЦИЯ ОБ АВТОРАХ [AUTHORS INFO]}

*Емельянов Андрей Олегович, к.м.н. [Andrey O. Emelyanov, PhD]; адрес: Россия, 117036, г. Москва, ул. Дм. Ульянова, д. 11 [address: 11 Dm. Ulyanova str., 117036, Moscow, Russia]; ORCID: https://orcid.org/0000-0002-3742-0231; e-library SPIN: 8110-5540; e-mail: endiab@mail.ru

Лаптев Дмитрий Никитич, Д.м.н. [Dmitry N. Laptev, PhD]; ORCID: https://orcid.org/0000-0002-4316-8546; e-library SPIN: 2419-4019; e-mail: laptevdn@ya.ru Медведева Елена Джоновна [Elena D. Medvedeva, MD]; ORCID: https://orcid.org/0000-0001-6892-1963; e-library SPIN: 4829-2175; e-mail: ed-med-ved@yandex.ru Переверзева Светлана Владимировна [Svetlana V. Pereverzeva, MD]; ORCID: https://orcid.org/0000-0002-5104-7218; eLibrary SPIN: 3972-2831; e-mail: sesega.pere@gmail.com

Петеркова Валентина Александровна, д.М.Н., профессор, академик РАН [Valentina A. Peterkova, Phd, professor, academician of Russian Academy of Medical Sciences]; ORCID: https://orcid.org/0000-0002-5507-4627; e-library SPIN: 4009-2463; e-mail: peterkovava@hotmail.com

\section{ЦИТИРОВАТЬ:}

Лаптев Д.Н., Емельянов А.О., Медведева Е.Д., Переверзева С.В., Петеркова В.А. Длительный гликемический контроль и факторы, ассоциированные с ответом на помповую инсулинотерапию у детей // Сахарный диабет. - 2021. T. 24. — №2. — C. 122-132. doi: https://doi.org/10.14341/DM12530

\section{TO CITE THIS ARTICLE:}

Laptev DN, Emelyanov AO, Medvedeva ED, Pereverzeva SV, Peterkova VA. Long-term glycemic control and factors, associated with response to pump insulin therapy in children. Diabetes Mellitus. 2021;24(2):122-132. doi: https://doi.org/10.14341/DM12530 